\title{
ON $\Lambda$-CONVERGENCE ALMOST EVERYWHERE OF MULTIPLE TRIGONOMETRIC FOURIER SERIES ${ }^{1}$
}

\author{
Nikolay Yu. Antonov \\ Krasovskii Institute of Mathematics and Mechanics, \\ Ural Branch of the Russian Academy of Sciences; \\ Ekaterinburg, Russia \\ Nikolai.Antonov@imm.uran.ru
}

\begin{abstract}
We consider one type of convergence of multiple trigonometric Fourier series intermediate between the convergence over cubes and the $\lambda$-convergence for $\lambda>1$. The well-known result on the almost everywhere convergence over cubes of Fourier series of functions from the class $L\left(\ln ^{+} L\right)^{d} \ln ^{+} \ln ^{+} \ln ^{+} L\left([0,2 \pi)^{d}\right)$ has been generalized to the case of the $\Lambda$-convergence for some sequences $\Lambda$.
\end{abstract}

Key words: Trigonometric Fourier series, Rectangular partial sums, Convergence almost everywhere.

Suppose that $d$ is a natural number, $\mathbb{T}^{d}=[-\pi, \pi)^{d}$ is a $d$-dimensional torus, and $\varphi: \quad[0,+\infty) \rightarrow$ $[0,+\infty)$ is a nondecreasing function. Let $\varphi(L)\left(\mathbb{T}^{d}\right)$ be the set of all Lebesgue measurable real-valued functions $f$ on the torus $\mathbb{T}^{d}$ such that

$$
\int_{\mathbb{T}^{d}} \varphi(|f(\mathbf{t})|) d \mathbf{t}<\infty .
$$

Let $f \in L\left(\mathbb{T}^{d}\right), \mathbf{k}=\left(k^{1}, k^{2}, \ldots, k^{d}\right) \in \mathbb{Z}^{d}, \mathbf{x}=\left(x^{1}, x^{2}, \ldots, x^{d}\right) \in \mathbb{R}^{d}$, and $\mathbf{k x}=k^{1} x^{1}+k^{2} x^{2}+\ldots+$ $k^{d} x^{d}$. Denote by

$$
c_{\mathbf{k}}=\frac{1}{(2 \pi)^{d}} \int_{\mathbb{T}^{d}} f(\mathbf{t}) e^{-i \mathbf{k t}} d \mathbf{t}
$$

the kth Fourier coefficient of the function $f$ and by

$$
\sum_{\mathbf{k} \in \mathbb{Z}^{d}} c_{\mathbf{k}} e^{i \mathbf{k x}}
$$

the multiple trigonometric Fourier series of the function $f$.

Let $\mathbf{n}=\left(n^{1}, n^{2}, \ldots, n^{d}\right)$ be a vector with nonnegative integer coordinates, and let $S_{\mathbf{n}}(f, \mathbf{x})$ be the $\mathbf{n}$ th rectangular partial sum of series (1):

$$
S_{\mathbf{n}}(f, \mathbf{x})=\sum_{\mathbf{k}=\left(k^{1}, \ldots, k^{d}\right):\left|k^{j}\right| \leq n^{j}, 1 \leq j \leq d} c_{\mathbf{k}} e^{i \mathbf{k x}} .
$$

Denote by mes $E$ the Lebesgue measure of a set $E$ and let $\ln ^{+} u=\ln (u+e), u \geq 0$.

In 1915, in the case $d=1$, N.N. Luzin (see [1]) suggested that the trigonometric Fourier series of any function from $L^{2}(\mathbb{T})$ converges almost everywhere. A.N. Kolmogorov [2] constructed an example of a function $F \in L(\mathbb{T})$ whose trigonometric series diverges almost everywhere and, later on [3], of a function from $L(\mathbb{T})$ with the Fourier series divergent everywhere on $\mathbb{T}$. L. Carleson [4] proved that Luzin's conjecture is true: if $f \in L^{2}(\mathbb{T})$, then the Fourier series of the function $f$ converges almost

\footnotetext{
${ }^{1}$ This work was supported by the Russian Science Foundation (project no. 14-11-00702).
} 
everywhere. R. Hunt [5] generalized the statement about the almost everywhere convergence of the Fourier series to the class $L\left(\ln ^{+} L\right)^{2}(\mathbb{T})$, particularly, to $L^{p}(\mathbb{T})$ with $p>1$. P. Sjölin [6] generalized it to the wider class $L\left(\ln ^{+} L\right)\left(\ln ^{+} \ln ^{+} L\right)(\mathbb{T})$. In [7], the author showed that the condition $f \in L\left(\ln ^{+} L\right)\left(\ln ^{+} \ln ^{+} \ln ^{+} L\right)(\mathbb{T})$ is also sufficient for the almost everywhere convergence of the Fourier series of the function $f$. At present, the best negative result in this direction belongs to S.V. Konyagin [8]: if a function $\varphi(u)$ satisfies the condition $\varphi(u)=o(u \sqrt{\ln u / \ln \ln u})$ as $u \rightarrow+\infty$, then, in the class $\varphi(L)(\mathbb{T})$, there exists a function with the Fourier series divergent everywhere on $\mathbb{T}$.

Let us now consider the case $d \geq 2$, i.e., the case of multiple Fourier series. Let $\lambda \geq 1$. A multiple Fourier series of a function $f$ is called $\lambda$-convergent at a point $\mathbf{x} \in \mathbb{T}^{d}$ if there exists a limit

$$
\lim _{\min \left\{n^{j}: 1 \leq j \leq d\right\} \rightarrow+\infty} S_{\mathbf{n}}(f, \mathbf{x})
$$

considered only for vectors $\mathbf{n}=\left(n^{1}, n^{2}, \ldots, n^{d}\right)$ such that $1 / \lambda \leq n^{i} / n^{j} \leq \lambda, \quad 1 \leq i, j \leq d$. The $\lambda$-convergence is called the convergence over cubes (the convergence over squares for $d=2$ ) in the case $\lambda=1$ and the Pringsheim convergence in the case $\lambda=+\infty$, i. e., in the case without any restrictions on the relation between coordinates of vectors $\mathbf{n}$.

N.R. Tevzadze [9] proved that, if $f \in L^{2}\left(\mathbb{T}^{2}\right)$, then the Fourier series of the function $f$ converges over cubes almost everywhere. Ch. Fefferman [10] generalized this result to functions from $L^{p}\left(\mathbb{T}^{d}\right)$, $p>1, d \geq 2$. P. Sjölin [11] showed that, if a function $f$ is from the class $L\left(\ln ^{+} L\right)^{d}\left(\ln ^{+} \ln ^{+} L\right)\left(\mathbb{T}^{d}\right)$, $d \geq 2$, then its Fourier series converges over cubes almost everywhere. The author [12] (see also [13]) proved the almost everywhere convergence over cubes of Fourier series of functions from the class $L\left(\ln ^{+} L\right)^{d}\left(\ln ^{+} \ln ^{+} \ln ^{+} L\right)\left(\mathbb{T}^{d}\right)$. The best current result concerning the divergence over cubes on a set of positive measure of multiple Fourier series of functions from $\varphi(L)\left(\mathbb{T}^{d}\right), d \geq 2$, belongs to S.V. Konyagin [14]: for any function $\varphi(u)=o\left(u(\ln u)^{d-1} \ln \ln u\right)$ as $u \rightarrow+\infty$, there exists a function $F \in \varphi(L)\left(\mathbb{T}^{d}\right)$ with the Fourier series divergent over cubes everywhere.

On the other hand, Ch. Fefferman [15] constructed an example of a continuous function of two variables, i. e., a function from $C\left(\mathbb{T}^{2}\right)$ whose Fourier series diverges in the Pringsheim sense everywhere on $\mathbb{T}^{2}$. M. Bakhbukh and E.M. Nikishin [16] proved that there exists $F \in C\left(\mathbb{T}^{2}\right)$ such that its modulus of continuity satisfies the condition $\omega(F, \delta)=O\left(\ln ^{-1}(1 / \delta)\right)$ as $\delta \rightarrow+0$ and its Fourier series diverges in the Pringsheim sense almost everywhere. A.N. Bakhvalov [17] established that, for $m \in \mathbb{N}$ and any $\lambda>1$, there is a function $F \in C\left(\mathbb{T}^{2 m}\right)$ such that the Fourier series of $F$ is $\lambda$-divergent everywhere and the modulus of continuity of $F$ satisfies the condition

$$
\omega(F, \delta)=O\left(\ln ^{-m}(1 / \delta)\right), \quad \delta \rightarrow+0 .
$$

Later on, Bakhvalov [18] proved the existence of a function $F \in C\left(\mathbb{T}^{2 m}\right)$ satisfying condition (2) and such that its Fourier series is $\lambda$-divergent for all $\lambda>1$ simultaneously.

Let $\Lambda=\left\{\lambda_{\nu}\right\}_{\nu=1}^{\infty}$ be a nonincreasing sequence of positive numbers. Assume that

$$
\Omega_{\Lambda}=\left\{\mathbf{n}=\left(n^{1}, n^{2}, \ldots, n^{d}\right) \in \mathbb{N}^{d}: \frac{1}{1+\lambda_{n^{i}}} \leq \frac{n^{i}}{n^{j}} \leq 1+\lambda_{n^{j}}, \quad 1 \leq i, j \leq d\right\} .
$$

We will say that a multiple Fourier series of a function $f \in L\left(\mathbb{T}^{d}\right)$ is $\Lambda$-convergent at a point $\mathbf{x} \in \mathbb{T}^{d}$ if there exists a limit

$$
\lim _{\mathbf{n} \in \Omega_{\Lambda}, \min \left\{n^{j}: 1 \leq j \leq d\right\} \rightarrow \infty} S_{\mathbf{n}}(f, \mathbf{x}) .
$$

Let us note that, if $\lambda_{\nu} \equiv \lambda-1$ for some $\lambda>1$, then the condition of $\Lambda$-convergence turns into the condition of $\lambda$-convergence defined above. And if $\lambda_{\nu} \rightarrow 0$ as $\nu \rightarrow \infty$, then the condition of $\Lambda$-convergence is weaker than the condition of $\lambda$-convergence for any $\lambda>1$. 
The author proved [19] that, if a sequence $\Lambda=\left\{\lambda_{\nu}\right\}_{\nu=1}^{\infty}$ satisfies the condition $\ln ^{2} \lambda_{\nu}=o(\ln \nu)$ as $\nu \rightarrow \infty$, then there exists a function $F \in C\left(\mathbb{T}^{2}\right)$ such that its Fourier series is $\Lambda$-divergent almost everywhere on $\mathbb{T}^{2}$.

In the present paper, we obtain the following statement that strengthens the result of [12].

Theorem 1. Assume that a nonincreasing sequence of positive numbers $\Lambda=\left\{\lambda_{\nu}\right\}_{\nu=1}^{\infty}$ satisfies the condition

$$
\lambda_{\nu}=O\left(\frac{1}{\nu}\right)
$$

and a function $\varphi:[0,+\infty) \rightarrow[0,+\infty)$ is convex on $[0,+\infty)$ and such that $\varphi(0)=0, \varphi(u) u^{-1}$ increases on $\left[u_{0},+\infty\right)$, and $\varphi(u) u^{-1-\delta}$ decreases on $\left[u_{0},+\infty\right)$ for some $u_{0} \geq 0$ and any $\delta>0$. Assume that the trigonometric Fourier series of any function $g \in \varphi(L)(\mathbb{T})$ converges almost everywhere on $\mathbb{T}$. Then, for any $d \geq 2$, the Fourier series of any function $f$ from the class $\varphi(L)\left(\ln ^{+} L\right)^{d-1}\left(\mathbb{T}^{d}\right)$ is $\Lambda$-convergent almost everywhere on $\mathbb{T}^{d}$.

Theorem 1 and the result of paper [7] imply the following statement.

Theorem 2. Let a nonincreasing sequence of positive numbers $\Lambda=\left\{\lambda_{\nu}\right\}_{\nu=1}^{\infty}$ satisfy condition (3), $d \geq 2$. Then the Fourier series of any function $f$ from the class

$$
L\left(\ln ^{+} L\right)^{d}\left(\ln ^{+} \ln ^{+} \ln ^{+} L\right)\left(\mathbb{T}^{d}\right)
$$

is $\Lambda$-convergent almost everywhere on $\mathbb{T}^{d}$.

P r o o f of Theorem 1. Let a sequence $\Lambda=\left\{\lambda_{\nu}\right\}_{\nu=1}^{\infty}$ and a function $\varphi$ satisfy the conditions of the theorem. Let $\varphi_{d}(u)=\varphi(u)\left(\ln ^{+} u\right)^{d-1}$ for short. Without loss of generality, we can consider only functions $\varphi_{d}$ such that the functions $\varphi_{d}(\sqrt{u})$ are concave on $[0,+\infty)$. Otherwise, we can consider the functions $\varphi_{d}\left(u+a_{d}\right)-b_{d}$ (with appropriate constants $a_{d}$ and $b_{d}$ ) instead of $\varphi_{d}$. The corresponding class $\varphi_{d}(L)\left(\mathbb{T}^{d}\right)$ will be the same in this case.

Denote by $S_{n}(f, \mathbf{x})$ the $n$th cubic partial sum of the Fourier series of the function $f$ :

$$
S_{n}(f, \mathbf{x})=S_{\mathbf{n}}(f, \mathbf{x}), \quad \text { where } \quad \mathbf{n}=(n, \ldots, n) .
$$

Suppose that

$$
\begin{aligned}
M(f, \mathbf{x}) & =\sup _{n \in \mathbb{N}}\left|S_{n}(f, \mathbf{x})\right|, \\
M_{\Lambda}(f, \mathbf{x}) & =\sup _{\mathbf{n} \in \Omega_{\Lambda}}\left|S_{\mathbf{n}}(f, \mathbf{x})\right| .
\end{aligned}
$$

Under the conditions of the theorem (see [12, formula (3.1) and Lemma 3]), there are constants $K_{d}>0$ and $y_{d} \geq 0$ such that

$$
\operatorname{mes}\left\{\mathbf{x} \in \mathbb{T}^{d}: M(f, \mathbf{x})>y\right\} \leq \frac{K_{d}}{y}\left(\int_{\mathbb{T}^{d}} \varphi_{d}(|f(\mathbf{x})|) d \mathbf{x}+1\right), \quad y>y_{d}, \quad f \in \varphi_{d}(L)\left(\mathbb{T}^{d}\right) .
$$

Using (4), we will prove that, for every $y>y_{d}$ and $f \in \varphi_{d}(L)\left(\mathbb{T}^{d}\right)$,

$$
\operatorname{mes}\left\{\mathbf{x} \in \mathbb{T}^{d}: M_{\Lambda}(f, \mathbf{x})>y\right\} \leq \frac{A_{d}}{y}\left(\int_{\mathbb{T}^{d}} \varphi_{d}(|f(\mathbf{x})|) d \mathbf{x}+1\right)
$$


and, for every $f \in \varphi_{d+1}(L)\left(\mathbb{T}^{d}\right)$,

$$
\int_{\mathbb{T}^{d}} M_{\Lambda}(f, \mathbf{x}) d \mathbf{x} \leq B_{d}\left(\int_{\mathbb{T}^{d}} \varphi_{d+1}(|f(\mathbf{x})|) d \mathbf{x}+1\right)
$$

where $A_{d}$ is independent of $f$ and $y ; B_{d}$ is independent of $f$.

The proof is by induction on $d$. Consider the base case, i. e., $d=1$ : statement (5) immediately follows from (4) because $M(f, \mathbf{x})=M_{\Lambda}(f, \mathbf{x})$ in the one-dimensional case. Similarly, (6) is a consequence of [5, Theorem 2].

Let $d \geq 2$. Suppose that statements (5) and (6) hold for $d-1$ and let us show that the same is true for $d$.

First, let us prove the validity of (5). Let $\mathbf{n}=\left(n^{1}, n^{2}, \ldots, n^{d}\right) \in \Omega_{\Lambda}$. According to (3), there is an absolute constant $C>0$ such that $\lambda_{\nu} \nu \leq C$ for all natural numbers $\nu$. Combining this with the definition of $\Omega_{\Lambda}$, we obtain that, for all $i, j \in\{1,2, \ldots, d\}$,

$$
\left|n^{i}-n^{j}\right| \leq C
$$

Recall that, if $\mathbf{n}=\left(n^{1}, n^{2}, \ldots, n^{d}\right)$, then the following representation holds for the $\mathbf{n}$ th rectangular partial sum of the Fourier series of the function $f$ :

$$
S_{\mathbf{n}}(f, \mathbf{x})=\frac{1}{\pi^{d}} \int_{\mathbb{T}^{d}} \prod_{j=1}^{d} D_{n^{j}}\left(t^{j}\right) f\left(x^{1}+t^{1}, \ldots, x^{d}+t^{d}\right) d t^{1} \ldots d t^{d}
$$

where $D_{n}(t)=\sin ((n+1 / 2) t) /(2 \sin (t / 2))$ is the one-dimensional Dirichlet kernel of order $n$. Let us add to and subtract from the $d$-dimensional Dirichlet kernel $\prod_{j=1}^{d} D_{n^{j}}\left(t^{j}\right)$ of order $\mathbf{n}$ the sum

$$
\sum_{k=2}^{d}\left(\prod_{j=1}^{k} D_{n^{1}}\left(t^{j}\right) \prod_{j=k+1}^{d} D_{n^{j}}\left(t^{j}\right)\right)
$$

(here and in what follows, we suppose that all products $\prod$ with an upper index less than a lower one are equal to 1). Rearranging the terms, we obtain

$$
\begin{aligned}
\prod_{j=1}^{d} D_{n^{j}}\left(t^{j}\right) & =\sum_{k=1}^{d-1}\left(\prod_{j=1}^{k} D_{n^{1}}\left(t^{j}\right) \prod_{j=k+1}^{d} D_{n^{j}}\left(t^{j}\right)-\prod_{j=1}^{k+1} D_{n^{1}}\left(t^{j}\right) \prod_{j=k+2}^{d} D_{n^{j}}\left(t^{j}\right)\right)+\prod_{j=1}^{d} D_{n^{1}}\left(t^{j}\right)= \\
= & \sum_{k=2}^{d}\left(\prod_{j=1}^{k-1} D_{n^{1}}\left(t^{j}\right) \prod_{j=k+1}^{d} D_{n^{j}}\left(t^{j}\right)\left(D_{n^{k}}\left(t^{k}\right)-D_{n^{1}}\left(t^{k}\right)\right)\right)+\prod_{j=1}^{d} D_{n^{1}}\left(t^{j}\right) .
\end{aligned}
$$


From this and (8), it follows that

$$
\begin{gathered}
S_{\mathbf{n}}(f, \mathbf{x})=\sum_{k=2}^{d} \frac{1}{\pi^{d}} \int_{\mathbb{T}^{d}}\left(\prod_{j=1}^{k-1} D_{n^{1}}\left(t^{j}\right) \prod_{j=k+1}^{d} D_{n^{j}}\left(t^{j}\right)\left(D_{n^{k}}\left(t^{k}\right)-D_{n^{1}}\left(t^{k}\right)\right)\right) \times \\
\times f\left(x^{1}+t^{1}, \ldots, x^{d}+t^{d}\right) d t^{1} \ldots d t^{d}+\frac{1}{\pi^{d}} \int_{\mathbb{T}^{d}} \prod_{j=1}^{d} D_{n^{1}}\left(t^{j}\right) f\left(x^{1}+t^{1}, \ldots, x^{d}+t^{d}\right) d t^{1} \ldots d t^{d}= \\
=\sum_{k=2}^{d} \frac{1}{\pi^{d}} \int_{\mathbb{T}}\left(D_{n^{k}}\left(t^{k}\right)-D_{n^{1}}\left(t^{k}\right)\right) \times \\
\times\left(\int_{\mathbb{T}^{d-1}} \prod_{j=1}^{k-1} D_{n^{1}}\left(t^{j}\right) \prod_{j=k+1}^{d} D_{n^{j}}\left(t^{j}\right) f\left(x^{1}+t^{1}, \ldots, x^{d}+t^{d}\right) d t^{1} \ldots d t^{k-1} d t^{k+1} \ldots d t^{d}\right) d t^{k}+S_{n^{1}}(f, \mathbf{x}) .
\end{gathered}
$$

Note that the latter term on the right hand side of (9) is the $n^{1}$ th cubic partial sum of the Fourier series of the function $f$. By (7), for all $k \in\{2,3, \ldots, d\}$ and $t \in \mathbb{T}$, we have $\left|D_{n^{k}}(t)-D_{n^{1}}(t)\right| \leq C$. Combining this with (9), we obtain

$$
\begin{gathered}
\left|S_{\mathbf{n}}(f, \mathbf{x})\right| \leq \sum_{k=2}^{d} \frac{C}{\pi^{d}} \int \mid \int_{\mathbb{T}} \prod_{\mathbb{T}^{d-1}}^{k-1} D_{n^{1}}\left(t^{j}\right) \prod_{j=k+1}^{d} D_{n^{j}}\left(t^{j}\right) \times \\
\times f\left(x^{1}+t^{1}, \ldots, x^{k-1}+t^{k-1}, t^{k}, x^{k+1}+t^{k+1}, \ldots, x^{d}+t^{d}\right) d t^{1} \ldots d t^{k-1} d t^{k+1} \ldots d t^{d}\left|d t^{k}+\right| S_{n^{1}}(f, \mathbf{x}) \mid .
\end{gathered}
$$

Applying the definitions of $M_{\Lambda}(f, \mathbf{x})$ and $M(f, \mathbf{x})$, from the latter estimate, we obtain

$$
\begin{gathered}
M_{\Lambda}(f, \mathbf{x}) \leq M(f, \mathbf{x})+\frac{C}{\pi} \sum_{k=2}^{d} \int_{\mathbb{T}} \sup _{\mathbf{n}=\left(n^{1}, n^{2}, \cdots, n^{d}\right) \in \Omega_{\Lambda}} \mid \frac{1}{\pi^{d-1}} \int_{\mathbb{T}^{d-1}} \prod_{j=1}^{k-1} D_{n^{1}}\left(t^{j}\right) \prod_{j=k+1}^{d} D_{n^{j}}\left(t^{j}\right) \times \\
\times f\left(x^{1}+t^{1}, \ldots, x^{k-1}+t^{k-1}, t^{k}, x^{k+1}+t^{k+1}, \ldots, x^{d}+t^{d}\right) d t^{1} \ldots d t^{k-1} d t^{k+1} \ldots d t^{d} \mid d t^{k}= \\
=M(f, \mathbf{x})+\frac{C}{\pi} \sum_{k=2}^{d} M_{k}(f, \mathbf{x}),
\end{gathered}
$$

where $M_{k}(f, \mathbf{x})$ denotes the $k$ th term of the sum on the left hand side of the equality in (10). Let $k \in\{2,3, \ldots, d\}$. Consider $M_{k}(f, \mathbf{x})$. Denote by $g_{k, t^{k}}$ the function of $d-1$ variables that can be obtained from the function $f$ by fixing the $k$ th variable $t^{k}$ :

$g_{k, t^{k}}\left(t^{1}, \ldots, t^{k-1}, t^{k+1}, \ldots, t^{d}\right)=f\left(t^{1}, \ldots, t^{k-1}, t^{k}, t^{k+1}, \ldots, t^{d}\right), \quad\left(t^{1}, \ldots, t^{k-1}, t^{k+1}, \ldots, t^{d}\right) \in \mathbb{T}^{d-1}$.

Define $\Omega_{\Lambda}^{\prime}$ as the set of $\mathbf{m}_{k}=\left(m^{1}, \ldots, m^{k-1}, m^{k+1}, \ldots, m^{d}\right) \in \mathbb{N}^{d-1}$ such that $\mathbf{m}=\left(m^{1}, \ldots, m^{d}\right) \in$ $\Omega_{\Lambda}$. Note that, in view of the invariance of $\Omega_{\Lambda}$ with respect to a rearrangement of variables, the set $\Omega_{\Lambda}^{\prime}$ is independent of $k$. Suppose that $\mathbf{n}_{k}^{\prime}=\left(n^{1}, \ldots, n^{1}, n^{k+1}, \ldots, n^{d}\right) \in \mathbb{N}^{d-1}$. Then

$$
\begin{gathered}
\frac{1}{\pi^{d-1}} \int_{\mathbb{T}^{d-1}} \prod_{j=1}^{k-1} D_{n^{1}}\left(t^{j}\right) \prod_{j=k+1}^{d} D_{n^{j}}\left(t^{j}\right) \times \\
\times f\left(x^{1}+t^{1}, \ldots, x^{k-1}+t^{k-1}, t^{k}, x^{k+1}+t^{k+1}, \ldots, x^{d}+t^{d}\right) d t^{1} \ldots d t^{k-1} d t^{k+1} \ldots d t^{d}=
\end{gathered}
$$




$$
=S_{\mathbf{n}_{k}^{\prime}}\left(g_{k, t^{k}},\left(x^{1}, \ldots, x^{k-1}, x^{k+1}, \ldots, x^{d}\right)\right)
$$

and

$$
M_{k}(f, \mathbf{x})=\int_{\mathbb{T}} \sup _{\mathbf{n}_{k}^{\prime} \in \Omega_{\Lambda}^{\prime}}\left|S_{\mathbf{n}_{k}^{\prime}}\left(g_{k, x^{k}},\left(x^{1}, \ldots, x^{k-1}, x^{k+1}, \ldots, x^{d}\right)\right)\right| d x^{k} .
$$

Further,

$$
\begin{gathered}
\operatorname{mes}\left\{\mathbf{x} \in \mathbb{T}^{d}: M_{k}(f, \mathbf{x})>y\right\}=2 \pi \text { mes }\left\{\left(x^{1}, \ldots, x^{k-1}, x^{k+1}, \ldots, x^{d}\right) \in \mathbb{T}^{d-1}: M_{k}(f, \mathbf{x})>y\right\} \leq \\
\leq \frac{2 \pi}{y} \int_{\mathbb{T}^{d-1}} M_{k}(f, \mathbf{x}) d x^{1} \ldots d x^{k-1} d x^{k+1} \ldots d x^{d}= \\
=\frac{2 \pi}{y} \int_{\mathbb{T}^{d}} \sup _{\mathbf{n}_{k}^{\prime} \in \Omega_{\Lambda}^{\prime}}\left|S_{\mathbf{n}_{k}^{\prime}}\left(g_{k, x^{k}},\left(x^{1}, \ldots, x^{k-1}, x^{k+1}, \ldots, x^{d}\right)\right)\right| d \mathbf{x}= \\
=\frac{2 \pi}{y} \int_{\mathbb{T}}\left(\int_{\mathbb{T}^{d-1}} \sup _{\mathbf{n}_{k}^{\prime} \in \Omega_{\Lambda}^{\prime}}\left|S_{\mathbf{n}_{k}^{\prime}}\left(g_{k, x^{k}},\left(x^{1}, \ldots, x^{k-1}, x^{k+1}, \ldots, x^{d}\right)\right)\right| d x^{1} \ldots d x^{k-1} d x^{k+1} \ldots d x^{d}\right) d x^{k} .
\end{gathered}
$$

From this, applying the induction hypothesis (more precisely, statement (6) for the dimension $d-1$ ) to the inner integral on the right hand part of (11), we obtain

$$
\begin{aligned}
\operatorname{mes}\left\{\mathbf{x} \in \mathbb{T}^{d}: M_{k}(f, \mathbf{x})>y\right\} & \leq \frac{2 \pi}{y} \int_{\mathbb{T}}\left(B_{d-1} \int_{\mathbb{T}^{d-1}} \varphi_{d}(|f(\mathbf{x})|) d x^{1} \ldots d x^{k-1} d x^{k+1} \ldots d x^{d}+1\right) d x^{k} \leq \\
& \leq \frac{(2 \pi)^{2} B_{d-1}}{y}\left(\int_{\mathbb{T}^{d}} \varphi_{d}(|f(\mathbf{x})|) d \mathbf{x}+1\right) .
\end{aligned}
$$

According to (10),

$$
\left\{\mathbf{x} \in \mathbb{T}^{d}: M_{\Lambda}(f, \mathbf{x})>y\right\} \subset\left\{\mathbf{x} \in \mathbb{T}^{d}: M(f, \mathbf{x})>\frac{y}{2}\right\} \bigcup\left(\bigcup_{k=2}^{d}\left\{\mathbf{x} \in \mathbb{T}^{d}: M_{k}(f, \mathbf{x})>\frac{\pi y}{2(d-1) C}\right\}\right) .
$$

Combining (13), (4) and (12), we obtain (5) with the constant $A_{d}=2 K_{d}+8 \pi(d-1)^{2} B_{d-1} C$.

Now, we only need to prove the validity of statement (6). To this end, let us use statement (5) proved above.

From (5), it follows that the majorant $M_{\Lambda}(f, \mathbf{x})$ is finite almost everywhere on $\mathbb{T}^{d}$ for all $f \in$ $\varphi_{d}(L)\left(\mathbb{T}^{d}\right)$, in particular, for all $f \in L^{2}\left(T^{d}\right)$. Applying Stein's theorem on limits of sequences of operators $[20$, Theorem 1$]$, we see that the operator $M_{\Lambda}(f, \cdot)$ is of weak type $(2,2)$, i.e., there is a constant $A_{d}^{2}>0$ such that, for all $y>0$ and $f \in L^{2}\left(T^{d}\right)$,

$$
\operatorname{mes}\left\{\mathbf{x} \in \mathbb{T}^{d}: M_{\Lambda}(f, \mathbf{x})>y\right\} \leq \frac{A_{d}^{2}}{y^{2}} \int_{\mathbb{T}^{d}}|f(\mathbf{x})|^{2} d \mathbf{x} .
$$

Similarly, from [20, Theorem 3], we can obtain the following refinement of statement (5): there is a constant $\bar{A}_{d}>0$ such that, for all $y \geq \bar{y}_{d} / 2=\bar{A}_{d}$ and $f \in \varphi_{d}(L)\left(\mathbb{T}^{d}\right)$,

$$
\operatorname{mes}\left\{\mathbf{x} \in \mathbb{T}^{d}: M_{\Lambda}(f, \mathbf{x})>y\right\} \leq \int_{\mathbb{T}^{d}} \varphi_{d}\left(\frac{\bar{A}_{d}|f(\mathbf{x})|}{y}\right) d \mathbf{x} \leq \frac{\bar{A}_{d}}{y} \int_{\mathbb{T}^{d}} \varphi_{d}(|f(\mathbf{x})|) d \mathbf{x}
$$


Further, let $f \in \varphi_{d}(L)\left(\mathbb{T}^{d}\right)$ and $y>0$. Suppose that

$$
g(x)=g_{y}(x)=\left\{\begin{array}{ll}
f(x), & |f(x)|>y, \\
0, & |f(x)| \leq y
\end{array} \quad h(x)=h_{y}(x)=f(x)-g(x) .\right.
$$

Define $\lambda_{f}(y)=\operatorname{mes}\left\{\mathbf{x} \in \mathbb{T}^{d}: M_{\Lambda}(f, \mathbf{x})>y\right\}$. Then

$$
\lambda_{f}(y) \leq \operatorname{mes}\left\{\mathbf{x} \in \mathbb{T}^{d}: M_{\Lambda}(g, \mathbf{x})>y / 2\right\}+\operatorname{mes}\left\{\mathbf{x} \in \mathbb{T}^{d}: M_{\Lambda}(h, \mathbf{x})>y / 2\right\}=\lambda_{g}(y / 2)+\lambda_{h}(y / 2) .
$$

From this, using the equality

$$
\int_{\mathbb{T}^{d}} M_{\Lambda}(f, \mathbf{x}) d \mathbf{x}=-\int_{0}^{\infty} y d \lambda_{f}(y)=\int_{0}^{\infty} \lambda_{f}(y) d y
$$

(see, for example, [21, Chapter $1, \S 13$, formula (13.6)]), we obtain

$$
\int_{\mathbb{T}^{d}} M_{\Lambda}(f, \mathbf{x}) d \mathbf{x} \leq \bar{y}_{d}(2 \pi)^{d}+\int_{\bar{y}_{d}}^{\infty} \lambda_{f}(y) d y \leq \bar{y}_{d}(2 \pi)^{d}+\int_{\bar{y}_{d}}^{\infty} \lambda_{g}\left(\frac{y}{2}\right) d y+\int_{\bar{y}_{d}}^{\infty} \lambda_{h}\left(\frac{y}{2}\right) d y .
$$

Taking into account that $g \in \varphi_{d}(L)\left(\mathbb{T}^{d}\right)$ and $h \in L^{\infty}\left(\mathbb{T}^{d}\right) \subset L^{2}\left(\mathbb{T}^{d}\right)$ and applying estimate (15) to $\lambda_{g}(y / 2)$ and estimate (14) to $\lambda_{h}(y / 2)$, from (16), we obtain

$$
\begin{aligned}
& \int_{\mathbb{T}^{d}} M_{\Lambda}(f, \mathbf{x}) d \mathbf{x} \leq \bar{y}_{d}(2 \pi)^{d}+2 \bar{A}_{d} \int_{\bar{y}_{d}}^{\infty}\left(\frac{1}{y} \int_{\mathbb{T}^{d}} \varphi_{d}(|g(\mathbf{t})|) d \mathbf{t}\right) d y+4 A_{d}^{2} \int_{\bar{y}_{d}}^{\infty}\left(\frac{1}{y^{2}} \int_{\mathbb{T}^{d}}|h(\mathbf{t})|^{2} d \mathbf{t}\right) d y= \\
= & \bar{y}_{d}(2 \pi)^{d}+2 \bar{A}_{d} \int_{\bar{y}_{d}}^{\infty}\left(\frac{1}{y} \int_{\left\{\mathbf{t} \in \mathbb{T}^{d}:|f(\mathbf{t})|>y\right\}} \varphi_{d}(|f(\mathbf{t})|) d \mathbf{t}\right) d y+4 A_{d}^{2} \int_{\bar{y}_{d}}^{\infty}\left(\frac{1}{y^{2}} \int_{\left\{\mathbf{t} \in \mathbb{T}^{d}:|f(\mathbf{t})| \leq y\right\}}|f(\mathbf{t})|^{2} d \mathbf{t}\right) d y .
\end{aligned}
$$

Applying Fubibi's theorem to the integrals on the right hand side of (17), we conclude that

$$
\begin{gathered}
\int_{\mathbb{T}^{d}} M_{\Lambda}(f, \mathbf{x}) d \mathbf{x} \leq 2 \bar{A}_{d} \int_{\left\{\mathbf{t} \in \mathbb{T}^{d}:|f(\mathbf{t})|>\bar{y}_{d}\right\}} \varphi_{d}(|f(\mathbf{t})|)\left(\int_{\bar{y}_{d}}^{|f(\mathbf{t})|} \frac{d y}{y}\right) d \mathbf{t}+ \\
+4 A_{d}^{2} \int_{\mathbb{T}^{d}}|f(\mathbf{t})|^{2}\left(\int_{|f(\mathbf{t})|}^{\infty} \frac{d y}{y^{2}}\right) d \mathbf{t}+\bar{y}_{d}(2 \pi)^{d}
\end{gathered}
$$

hence, statement (6) follows easily.

Finally, the $\Lambda$-convergence of the Fourier series of an arbitrary function from the class $\varphi_{d}(L)\left(\mathbb{T}^{d}\right)$ can be obtained from (5) by means of standard arguments (see, for example, [12, Lemma 3]). Theorem 1 is proved. 


\section{REFERENCES}

1. Luzin N.N. Integral and trigonometric series. Moscow - Leningrad: GITTL. 1951. 550 p. [in Russian]

2. Kolmogoroff A. Une série de Fourier - Lebesgue divergente preque partout // Fund. math., 1923. Vol. 4. P. 324-328.

3. Kolmogoroff A. Une série de Fourier - Lebesgue divergente partout // C. r. Acad. sci. Paris, 1926. Vol. 183. P. 1327-1329.

4. Carleson L. On convergence and growth of partial sums of Fourier series // Acta math., 1966. Vol. 116, no. $1-2$. P. $135-157$. DOI: $10.1007 /$ BF02392815

5. Hunt R.A. On the convergence of Fourier series // Orthogonal expansions and their continuous analogues. SIU Press, Carbondale, Illinois, 1968. P. 235-255.

6. Sjölin P. An inequality of Paley and convergence a.e. of Walsh-Fourier series // Arkiv för mat., 1969. Vol. 7. P. 551-570. DOI: 10.1007/BF02590894

7. Antonov N.Yu. Convergence of Fourier series // East Journal on Approximations, 1996. Vol. 2, no. 2. P. $187-196$.

8. Konyagin S.V. On everywhere divergence of trigonometric Fourier series// Sb. Math., 2000. Vol. 191, no. 1. P. 97-120. DOI: 10.1070/SM2000v191n01ABEH000449

9. Tevzadze N.R. The convergence of the double Fourier series of a square integrable function // Soobshch. AN GSSR, 1970. Vol. 58, no. 2. P. 277-279. [in Russian]

10. Fefferman C. On the convergence of multiple Fourier series // Bull. Amer. Math. Soc., 1971. Vol. 77, no. 5. P. 744-745. DOI: 10.1090/S0002-9904-1971-12793-3

11. Sjölin P. Convergence almost everywhere of ertain singular integrals and multiple Fourier series // Arkiv för mat., 1971. Vol. 9, no. 1. P. 65-90. DOI: 10.1007/BF02383638

12. Antonov N.Yu. Almost everywhere convergence over cubes of multiple trigonometric Fourier series // Izv. Math., 2004. Vol. 68, no. 2, P. 223-241. DOI: 10.1070/IM2004v068n02ABEH000472

13. Antonov N.Yu. On the almost everywhere convergence of sequences of multiple rectangular Fourier sums // Proc. Steklov Inst. Math., 2009. Vol. 264, Suppl. 1. P. S1-S18. DOI: 10.1134/S0081543809050010

14. Konyagin S.V. On divergence of trigonometric Fourier series over cubes // Acta Sci. Math. (Szeged), 1995. Vol. 61. P. 305-329.

15. Fefferman C. On the divergence of multiple Fourier series // Bull. Amer. Math. Soc., 1971. Vol. 77, no. 2. P. 191-195. DOI: 10.1090/S0002-9904-1971-12675-7

16. Bakhbukh M., Nikishin E. M. The convergence of the double Fourier series of continuous functions // Siberian Math. Journal, 1973. Vol. 14, no. 6. P. 832-839. DOI: 10.1007/BF00975888

17. Bakhvalov A. N. Divergence everywhere of the Fourier series of continuous functions of several variables // Sb. Math., 1997. Vol. 188, no. 8. P. 1153-1170. DOI: 10.1070/SM1997v188n08ABEH000240.

18. Bakhvalov A. N. $\lambda$-divergence of the Fourier series of continuous functions of several variables // Mathematical Notes, 2002. Vol. 72, no. 3-4. P. 454-465. DOI: $10.4213 / \mathrm{mzm} 438$

19. Antonov N.Yu. On divergence almost everywhere of Fourier series of continuous functions of two variables // Izvestiya of Saratov University. New ser. Ser. Math. Mech. Inform., 2014. Vol. 14, iss. 4, pt. 2. P. 495-502. [in Russian]

20. Stein E.M. On limits of sequences of operators // Ann. Math., 1961. Vol. 74, no. 1. P. 140-170.

21. Zygmund A. Trigonometric series, vol. 1. New York: Cambridge Univ. Press, 1959. 383 p. 